\title{
CONSERVATION STATUS OF PLANTS AND HABITATS UNDER TEN YEARS OPERATION PHASE OF A WIND FARM. CASE STUDY: SFÂNTA ELENA KARST PLATEAU, IRON GATES NATURAL PARK, ROMANIA
}

\author{
Mihaela URZICEANU ${ }^{1,2}$, Paulina ANASTASIU ${ }^{1,2 *}$, Ioana-Minodora SÎRBU ${ }^{1,2}$, Tatiana Eugenia ȘESAN \\ ${ }^{1}$ University of Bucharest, Faculty of Biology, Independenţei Blvd. 91-95, 050095-Bucharest, Romania \\ ${ }^{2}$ University of Bucharest, Botanic Garden 'D. Brandza”, Şos. Cotroceni 32, 060114-Bucharest, Romania \\ ${ }^{3}$ Academy of Agricultural Sciences and Forestry, Mărăști Blvd. 61, 011464-Bucharest, Romania \\ email: paulina.anastasiu@bio.unibuc.ro
}

\begin{abstract}
Located in the Iron Gates Natural Park, Romania, at the foot of the Locvei Mountains, the Sfânta Elena Karst Plateau is a very picturesque territory, characterized by a karst relief, covered by High Nature Value grasslands. In the last decade this territory has been subjected to increasing anthropogenic pressures, and specific concerns on biodiversity have been raised after the construction of a wind farm in 2011. Starting from a concern expressed by a scientist, our study aims to provide current data on plant species with conservation value and to assess the conservation status of orchid-rich grasslands around wind turbines after ten years of wind farm operation. During the operation period we identified 19 plant taxa with conservation value, eight of which which are orchid species. Four of them are new reports for this territory: Gymnadenia conopsea, Neotinea ustulata, Neottia nidus-avis and Orchis simia, as well other rare taxa such as Cirsium grecescui, Lathyrus sphaericus, Linum hologynum and Rumex thyrsiflorus, are mentioned for the first time. The orchid-rich grasslands in the wind farm area correspond to the Natura 2000 habitat 6210* whose structure and floristic composition are within the acceptable limits of Favourable conservation status. The conservation status of plant species is directly dependent on the quality of their habitat, as well as the variety of pressure and threat factors in the area.
\end{abstract}

Keywords: grasslands, orchids, rare plants, wind turbines, pressure factors, protected areas

\section{Introduction}

The Sfânta Elena Karst Plateau (SEKP) is located in the Iron Gates Natural Park, at the foot of the Locvei Mountains, around Sfânta Elena village, Coronini commune (named Pescari in 1964-1995), Caraş Severin County, Romania (Fig. 1). It stretches between the Liuborajdea Valley and the Danube, in a hilly landscape, at altitudes between 200 and $600 \mathrm{~m}$ [39]. The relief is characterized by the presence of surface karst forms (sinkholes, dry karst valleys and limestone pavement), as well as deep karst forms, of which the best known is the Gaura cu Muscă Cave [53]. This relief, the cultural history of the old Czech community from Sfânta Elena village [32], and the natural habitats mixed with small-scale arable cultivation have created here an example of a man-made cultural landscape [56].

On the calcareous substrate, specific to the area, grasslands of High Nature Value grow in association with hilly forests and coppices consisting of species such as Acer campestre, Acer pseudoplatanus, Carpinus orientalis, Cotinus coggygria, Fraxinus ornus, Quercus spp., Syringa vulgaris and Tilia tomentosa $[39,52]$. Also, in this territory an interesting situation is an inversion 
of forest vegetation due to the climatic conditions back in the Atlantic Period (warmest period of the Holocene), maintained today by the Mediterranean character of the local climate, as here we can find at the lowest altitude in the country the presence of beech (Fagus sylvatica) [41, 8]. Thus, nowadays it can be observed, in a low-lying area from an altitudinal point of view, a mixture of boreal and montane flora together with species of Mediterranean origin [39].

Regarding the flora and vegetation, very few studies have been conducted on the grasslands of the SEKP, most of them focusing on the rocky area along the Danube, with insufficient data distribution. The first mentions regarding the local flora were made by Borza [3] from the Coronini Cave. In the biogeographical considerations on the Danube Gorge, Călinescu and Iana [5] indicate some plants from Coronini. Later, Păun et al. [44, 45] published their research on the flora and vegetation of the Berzeasca-Coronini sector, including chorological references for the Coronini area. Flora and vegetation of the Locvei Mountains is presented in detail in Coste's doctoral thesis [13], but the author does not refer to the territory of SEKP. Grigore and Coste [27] published plant associations on the Moldova Veche - Coronini sector, without giving the precise location, except for the plant association Syringo vulgaris - Carpinetum orientalis which is mentioned on slopes with calcareous substrate from Coronini. Some mentions for Coronini commune are also presented in the volumes of Flora României (red. T. Săvulescu): Beldie [2], Prodan [47], Nyárády [38], Morariu [33, 34, 35], Paucă [43], Grințescu I [28], Todor [58], Prodan and Nyárády [48], Ghișa [25], Dobrescu [17]. Also, in the study about the fungi of the Danube Gorge, Sandu-Ville et al. [49] present some host plants in the Coronini area. Many chorological data on the flora and vegetation of Iron Gates Natural Park can be found in Sorina Matacă's doctoral thesis [30], but for SEKP there are floristic mentions mainly on the rocks along the Danube and Gaura cu Muscă Cave. Also, some plants with conservation value in the area of SEKP are known from chorology papers [46], Comănescu and Ștefănuț [10], Anastasiu [1]. Thus, so far from SEKP are known about 65 taxa with conservative value, reported mainly from the rocks of the Danube Gorge.

The conservation importance of the SEKP is underlined by Milanovici [31] in an extensive study targeting orchids from the southern Banat, in which six orchid species are mentioned for the grasslands of the SEKP (Anacamptis coriophora, Anacamptis morio, Cephalanthera longifolia, Neottia ovata, Orchis mascula subsp. speciosa and Platanthera chlorantha). Due to the importance of its orchid-rich grasslands, Milanovici [31] recommends that SEKP should be included in the integral protection zone of the Iron Gates Natural Park.

In the last decade, a large part of this territory was subject to anthropogenic pressure, mainly since 2011 when the construction began of a wind farm with 21 wind turbines. This activity was described as one of the manifestations of the globalization tendencies in Sfânta Elena territory [56]. The wind farm construction produced environmental changes, influencing both the landscape and the land cover. New access roads and platforms around the wind turbines were created instead of arable land, grassland and scrub. Milanovici [31] considered this wind farm an important negative factor on the orchid grasslands on SEKP, wondering itself "what impact will the wind turbines have on grasslands?". Also, given the fact that the wind turbines interfere with the picturesque landscape of the area, some authors were concerned about the risk of negative influence on tourism and the landscape of the Iron Gates Natural Park and the Natura 2000 Network [29, 56].

Based on the concern expressed by Milanovici [31], our study aims to provide current data on plant species of conservation value and to assess the conservation status of orchid-rich 
grasslands around wind turbines after ten years of wind farm operation.

\section{Material and Methods}

\section{Investigated area}

The investigated area is represented by the "Sfânta Elena" wind farm which consists of 21 wind turbines (henceforth being noted as WT). The wind farm is located at a distance of $c .600 \mathrm{~m}$ from the Danube, some $500 \mathrm{~m}$ from the St. Elena village and $600 \mathrm{~m}$ from the Coronini village (Fig. 1). The 21 WTs are located in a variety of habitats and vegetation types: arable lands, forests, bushes and grasslands. The area occupied by a WT consists of a cement pad around the tower, an access road and an area cleared of vegetation during the construction period, which is referred to as the technological platform.

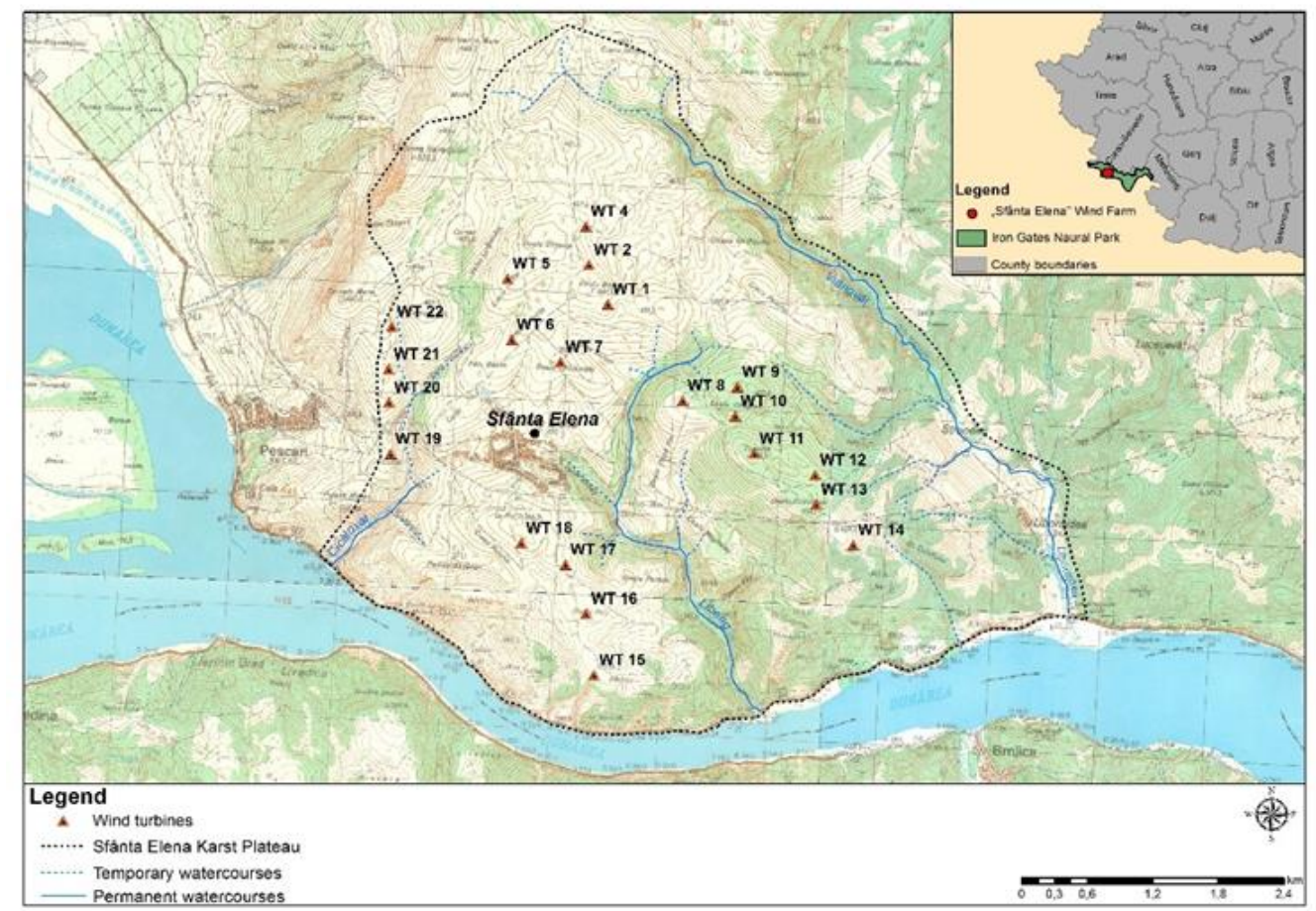

Fig. 1: Map of the Sfânta Elena Karst Plateau with the 21 WT (compiled after Oancea et al. 1987)

\section{Data collection and analysis}

Field surveys were conducted monthly in 2017-2020, during the vegetation period (MarchSeptember). We investigated the plants of conservative value in two types of 2,500 $\mathrm{m}^{2}$ established plots: 1) with vegetation affected by construction works corresponding to a technological platform and 2) with vegetation unaffected by construction works corresponding to habitats in the adjacent area. For unaffected plots we surveyed the floristic composition, which we used as support in identifying grassland habitats with conservation value.

We used the concept of "conservation value" in accordance with the significance of Capmourteres and Anand [6], referring to the plants and habitats listed in at least one of the National Red Lists [40, 16, 4], the National Red Book [15] or Habitats Directive [14].

For each plant taxa of conservation value around wind turbines we provide data regarding the WT number and the corresponding GPS coordinates, the habitat and the plots type in which the species were identified, as well as previous literature reporting of the species in the area. The distribution data are presented in the form of maps processed in the ArcGIS 10.4 software [19]. 
We calculated the number of plant taxa of conservation value around each WT and their frequency in the analysed areas (affected vs unaffected), to identify the areas with the highest conservation value. The frequency is given as a percentage and indicates how often the plants appear in the analysed plots, being calculated according to the formula $F=n / N x 100$, where $n$ is the number of plots containing plant taxa with conservation value, and $N$ is the total number of plots investigated.

The conservation status of the plants discussed in this study was evaluated based on a threelevel scale (Favourable; Unfavourable-Inadequate, Unfavourable-Bad) [11], by taking into consideration the following parameters: habitat quality, size of the species population in the plot, respectively abundance/ dominance (AD) score and the significance of pressures. To determine the $\mathrm{AD}$ score we used Braun-Blanquet scale: $+=$ few individuals, low cover; $1=$ abundant individuals with reduced cover or few individuals with greater cover (up to $1 / 10$ of the surface); $2=$ very abundant individuals or with a cover of $1 / 10-1 / 4$ of the surface; $3=$ cover $1 / 4-1 / 2$ of the surface, irrespective of the number of individuals; $4=$ cover $1 / 2-3 / 4$ of the surface, irrespective of the number of individuals; $5=$ high cover of $3 / 4$ of the surface, irrespective of the number of individuals; $r=1$ 5 individuals with negligible cover.

The nomenclature is in accordance with Euro+Med PlantBase database [20] and Sârbu et al. [51].

The identification of the grassland habitats was made based on the characteristic and dominant species, in accordance with the Interpretation Manual of Natura 2000 Habitats in Romania [24] and the EUR28 Manual [12].

In order to assess the conservation status of the grassland habitats of community interest, the conditions for Favourable conservation status were analysed according to the synthesis paper Habitat fact-sheets [36].

\section{Results and Discussion}

Around all 21 wind turbines we registered 19 plant taxa of conservation value (Table 1), of which eight are orchid species (Anacamptis coriophora, Anacamptis morio, Gymnadenia conopsea, Neotinea ustulata, Neottia nidus-avis, Neottia ovata, Orchis mascula subsp. speciosa, and Orchis simia). Thus, the Orchidaceae family is the best represented among the taxa of conservation value, followed by the Compositae family with two taxa (Cirsium grecescui and Echinops bannaticus), the other families being represented by only one taxon each (Caryophyllaceae, Fabaceae, Plantaginaceae, Linaceae, Asparagaceae, Poaceae, Polygonaceae, Apiaceae, and Lamiaceae) (Table 1).

Also, the total of 19 taxa includes eight newly observed taxa not previously mentioned in the scientific literature for this territory: Cirsium grecescui, Gymnadenia conopsea, Lathyrus sphaericus, Linum hologynum, Neotinea ustulata, Neottia nidus-avis, Orchis simia, and Rumex thyrsiflorus. With the exception of Rumex thyrsiflorus, all these species are known from the literature to be present in the Iron Gates Natural Park, in adjacent areas to the SEKP [30, 31]. Thus, Cirsium grecescui is distributed in the sectors Ogradena-Eșelnița, Ilovița-Vârciorova, Gura Vârciorova - Gura Văii, Cazanele Mici [30], Gymnadenia conopsea is distributed in Baziaș, Radimna Valley, Micoş Valley, Belobreşca, Eşelniţa, Ogradena, Orşova [31], Lathyrus sphaericus is distributed in Baziaș, Pojejena-Măcești-Moldova Veche, Moldova Nouă, Golovârf Hill, SvinițaTricule, Cazanele Mici, Valea Mraconiei, Orșova, Gura Văii-Schela Cladovei [30], Linum 
hologynum is distributed in Svinița, Mehedinți Plateau-Duhovnei Hill [30], Neotinea ustulata is distributed in Radimna Valley (Zăvoi), Moldova Nouă [13], Neottia nidus-avis is distributed in Baziaș, Baziaş - Pojejena, Valea Radimna, Valea Micloş, Moldova Nouă, Valea Mare - Moldova Nouă, Belobreşca, Movita, Velika Lokva, Divici - Ribiş, Velika Reka, Şuşca - Poiana Lisa, Valea Mraconiei, Valea Eşelniţei, Valea Țarovăţ, Tisoviţa, Plavişeviţa, Valea Mare - Moldova Nouă, Valea Şirinea [31], Orchis simia is distributed in Baziaş, Divici, Vârciorova, Gura Slătinicului and Cracul Ciolane, Gura Văii on Cârlan Hill, Oglănic Forest, Valea Mraconiei, Belobreşca, Ribiş - Divici, Orşova, Vârciorova, Slătinic, Valea Oglănicului [31].

The presence of Rumex thyrsiflorus on the SEKP is the first report in the botanical literature, but in a zoological paper Trnka et al. [59] mention Rumex thyrsiflorus as a host plant for the beetle (weevil) species Lixus neglectus, present in this area. Rumex thyrsiflorus was mentioned before in Romania from the counties of Bihor [9, 57], Bistrița-Năsăud, Brașov, Cluj, Galați, Maramureș, Vaslui [15, 51], Mehedinți [7], Satu-Mare [57] and Sălaj [37].

Regarding the sozological status (Table 1), most of the taxa are listed as Rare plants in at least one of the National Red Lists. A special situation occurs in the case of the species Agrostemma githago and Linaria arvensis, which, although listed in the Red Lists, are considered alien plants (with archaeophytic character) in our country [55]. The species Agrostemma githago has a Vulnerable status in the Red List of Boșcaiu et al. [4] and can rarely be seen in our country [51, 55]. Although it was an abundant plant in arable crops during the mid-20th century, due to seed cleaning and modern agricultural practices, this species has been placed on the Red Lists of many European countries [22, 18]. The presence of Agrostemma githago in the SEKP, even if it has a small population (2-3 individuals/ plot around WT), can be explained by the specifics of agricultural workings practiced on a small scale, poorly mechanized and with organic fertilizers [50]. The species Linaria arvensis is listed in the Red List of Dihoru and Dihoru [16] as a plant for which there is insufficient information on its inclusion in Endemic, Vulnerable or Rare categories. It has a sporadic presence in our country, with preferences for ruderal, cultivated places [51], cited from Cluj, Mureş, Harghita, Braşov, Alba, Iaşi, Suceava, Tulcea counties [55], and most recently reported by Anastasiu [1] from both Hunedoara and Caraş-Severin counties.

Only two of the taxa are listed in the National Red Book [15], namely Orchis simia with Endangered (EN) status and Rumex thyrsiflorus with, respectively, Critically Endangered (CR) status (Table 1).

Around each of the wind turbines, except WT 5, we identified at least one or more plant taxa with conservation value, in various types of habitats (Table 2, Fig. 2). The plant taxa with conservation value that we report for the first time are distributed around 16 of the 21 turbines (Fig. 3).

The most numerous plant taxa of conservation value (six taxa) have been identified around WT 4 which is surrounded by grassland habitat. Of these six taxa, five are orchid species (Anacamptis coriophora, Anacamptis morio, Gymnadenia conopsea, Neotinea ustulata and Orchis mascula subsp. speciosa), and the sixth is the Critically Endangered species Rumex thyrsiflorus, which gives the conservation value to this habitat area. Numerous plant taxa with conservation value have also been found around the WT 12 which is surrounded by a broad-leaved forest habitat (four taxa, of which two are orchids, Neottia nidus-avis and Neottia ovata), and also around the WT 15 and WT 16, which are surrounded by arable land with some areas of natural vegetation (four taxa each). Also, around the turbines surrounded by grassland areas mixed with shrubs (WT 
20, WT 21, WT 22) we identified a high number of taxa (three different species at each turbine), including the orchid species Anacamptis morio and Orchis simia (Fig. 2).

Table 1: Plant taxa with conservation value inventoried around wind turbines

\begin{tabular}{|c|c|c|c|c|c|c|}
\hline No. & Taxa & Family & NRB [15] & NRL [4] & NRL [16] & $\begin{array}{l}\text { NRL } \\
{[40]}\end{array}$ \\
\hline 1 & Agrostemma githago $\mathrm{L}$. & Caryophyllaceae & & V & & \\
\hline 2 & $\begin{array}{l}\text { Anacamptis coriophora }(\mathrm{L} .) \\
\text { R. M. Bateman, Pridgeon \& } \\
\text { M. W. Chase }\end{array}$ & Orchidaceae & & & & $\mathrm{R}$ \\
\hline 3 & $\begin{array}{l}\text { Anacamptis morio (L.) R. M. } \\
\text { Bateman, Pridgeon \& M. W. } \\
\text { Chase }\end{array}$ & Orchidaceae & & & & $\mathrm{R}$ \\
\hline 4 & \#Cirsium grecescui Rouy & Compositae & & & $\mathrm{R}$ & $\mathrm{R}$ \\
\hline 5 & Echinops bannaticus Schrad. & Compositae & & & $\mathrm{R}$ & $\mathrm{R}$ \\
\hline 6 & $\begin{array}{l}\text { \#Gymnadenia conopsea (L.) } \\
\text { R. Br. }\end{array}$ & Orchidaceae & & $\mathrm{R}$ & & $\mathrm{R}$ \\
\hline 7 & \#Lathyrus sphaericus Retz. & Fabaceae & & $\mathrm{R}$ & $\mathrm{R}$ & \\
\hline 8 & Linaria arvensis (L.) Desf. & Plantaginaceae & & & I & \\
\hline 9 & \#Linum hologynum Rchb. & Linaceae & & & $\mathrm{R}$ & $\mathrm{R}$ \\
\hline 10 & $\begin{array}{l}\text { Muscari neglectum Guss. ex } \\
\text { Ten. }\end{array}$ & Asparagaceae & & & & $\mathrm{R}$ \\
\hline 11 & $\begin{array}{l}\text { \#Neotinea ustulata (L.) R. M. } \\
\text { Bateman, Pridgeon \& M. W. } \\
\text { Chase }\end{array}$ & Orchidaceae & & & & $\mathrm{R}$ \\
\hline 12 & \#Neottia nidus-avis (L.) Rich. & Orchidaceae & & & & $\mathrm{R}$ \\
\hline 13 & $\begin{array}{l}\text { Neottia ovata (L.) Bluff \& } \\
\text { Fingerh. }\end{array}$ & Orchidaceae & & & & $\mathrm{R}$ \\
\hline 14 & $\begin{array}{l}\text { Orchis mascula subsp. } \\
\text { speciosa (Mutel) Hegi }\end{array}$ & Orchidaceae & & & & $\mathrm{R}$ \\
\hline 15 & \#Orchis simia Lam. & Orchidaceae & EN & $\mathrm{R}$ & $\mathrm{R}$ & $\mathrm{R}$ \\
\hline 16 & $\begin{array}{l}\text { Piptatherum virescens (Trin.) } \\
\text { Boiss. }\end{array}$ & Poaceae & & & & $\mathrm{R}$ \\
\hline 17 & \#Rumex thyrsiflorus Fingerh. & Polygonaceae & $\mathrm{CR}$ & $\mathrm{R}$ & I & $\mathrm{R}$ \\
\hline 18 & Smyrnium perfoliatum $\mathrm{L}$. & Apiaceae & & & $\mathrm{R}$ & $\mathrm{R}$ \\
\hline 19 & Teucrium montanum $\mathrm{L}$. & Lamiaceae & & & $\mathrm{R}$ & \\
\hline
\end{tabular}

\# New taxa, not previously reported in the scientific botanical literature for SEKP

$\mathrm{NRB}=$ National Red Book, NRL $=$ National Red List, $\mathrm{VU}=$ Vulnerable, $\mathrm{EN}=$ Endangered, $\mathrm{CR}=$ Critically Endangered, $\mathrm{R}=$ Rare, $\mathrm{V}=$ Vulnerable, $\mathrm{I}=$ Indeterminate.

In terms of frequency, the most frequent plant species were observed in both affected and unaffected plots (Table 3). The species Cirsium grecescui, Lathyrus sphaericus, and Agrostemma githago have a higher frequency in the affected plots than in the unaffected plots, this situation being associated with the presence of the arable land around turbines (Table 2). This may be due to the preferences of these species for ruderal, or segetal places to the detriment of natural ones [51]. Also, the orchid species Anacamptis morio occurs in affected plots, but with a lower frequency than in the unaffected ones $(19.05 \% / 9.52 \%)$. The majority of the other plant species has a lower frequency, being present mostly in unaffected plots (Muscari neglectum, Smyrnium perfoliatum, Piptatherum virescens, Linum hologynum, Anacamptis coriophora, Neotinea ustulata, Neottia nidus-avis, Neottia ovata, Orchis mascula subsp. speciosa, Orchis simia, Rumex thyrsiflorus, Teucrium montanum and Gymnadenia conopsea). We specify that Orchis mascula 
subsp. speciosa was identified on an unaffected plot (corresponding to a nearby grassland), but at more than $100 \mathrm{~m}$ from the WT 4.

The high frequency of plant species present in the affected areas shows their good adaptability over time to the revegetation process. This is the case with the species Anacamptis morio, which has spread its population from the unaffected grasslands to the WT 2 and WT 4 technological platforms.

Regarding previous mentions in the literature (Table 2), we found little information related to the spot distribution, population size or conservation status of the taxa analysed. The orchid Anacamptis coriophora is mentioned by Păun et al. [44] on the area of the Coronini commune and by Milanovici [31] on the grasslands of Sfânta Elena. Milanovici [31] points out the presence of the orchids Anacamptis coriophora, Anacamptis morio, Neottia ovata, and Orchis mascula subsp. speciosa in the grasslands of SEKP, but the population size and the conservation status are presented for the entire Iron Gates Natural Park (Anacamptis coriophora-over 3,000 individuals, with relatively good conservation status, Anacamptis morio - over 15,000 individuals, with good conservation status, Neottia ovata - less than 1,000 individuals and Orchis mascula subsp. speciosa - over 3,000 individuals, both with relatively good conservation status). The other taxa identified around the wind turbines were previously reported either from the rocky areas of Coronini (Echinops bannaticus and Piptatherum virescens), the Coronini commune (Smyrnium perfoliatum and Teucrium montanum) or from area sectors that include the commune of Coronini (Agrostemma githago and Muscari neglectum). Also, for none of these taxa did we find any information other than their presence in the area.

Table 2: Distribution of plant taxa with conservation value around wind turbines and previous reportings in the area

\begin{tabular}{|c|c|c|c|c|c|}
\hline Taxon name & $\begin{array}{l}\text { Wind } \\
\text { turbines }\end{array}$ & Coordinates & $\begin{array}{l}\text { Habitat type } \\
\text { around wind } \\
\text { turbine }\end{array}$ & Type area & $\begin{array}{c}\text { Previous } \\
\text { reportings in the } \\
\text { area }\end{array}$ \\
\hline \multirow[t]{4}{*}{ Agrostemma githago } & WT 6 & $\begin{array}{l}44^{\circ} 41^{\prime} 13.87^{\prime \prime} \mathrm{N} / \\
21^{\circ} 42^{\prime} 38.73^{\prime \prime} \mathrm{E}\end{array}$ & Arable land & Affected & \multirow[t]{4}{*}{$\begin{array}{l}\text { Moldova Veche - } \\
\text { Coronini [27] }\end{array}$} \\
\hline & WT 7 & $\begin{array}{l}44^{\circ} 41^{\prime} 7.93^{\prime \prime} \mathrm{N} \\
21^{\circ} 42^{\prime} 59.47^{\prime \prime} \mathrm{E}\end{array}$ & Arable land & Affected & \\
\hline & WT 15 & $\begin{array}{l}44^{\circ} 39^{\prime} 35.46^{\prime \prime} \mathrm{N} / \\
21^{\circ} 43^{\prime} 18.61^{\prime \prime} \mathrm{E}\end{array}$ & $\begin{array}{l}\text { Arable land with } \\
\text { areas of natural } \\
\text { vegetation }\end{array}$ & $\begin{array}{l}\text { Affected + } \\
\text { Unaffected }\end{array}$ & \\
\hline & WT 16 & $\begin{array}{l}44^{\circ} 39^{\prime} 53.55^{\prime \prime} \mathrm{N} / \\
21^{\circ} 43^{\prime} 14.26^{\prime \prime} \mathrm{E}\end{array}$ & Arable land & Unaffected & \\
\hline $\begin{array}{l}\text { Anacamptis } \\
\text { coriophora }\end{array}$ & WT 4 & $\begin{array}{l}44^{\circ} 41^{\prime} 48.40^{\prime \prime} \mathrm{N} / \\
21^{\circ} 43^{\prime} 7.70^{\prime \prime} \mathrm{E}\end{array}$ & $\begin{array}{l}\text { Grassland } \\
\left(6210^{*}\right)\end{array}$ & Unaffected & $\begin{array}{l}\text { Coronini [44], } \\
\text { Sfânta Elena [31] }\end{array}$ \\
\hline \multirow[t]{6}{*}{ Anacamptis morio } & WT 1 & $\begin{array}{l}44^{\circ} 41 ' 25.62^{\prime \prime} \mathrm{N} / \\
21^{\circ} 43^{\prime} 18.09^{\prime \prime} \mathrm{E}\end{array}$ & Arable land & Unaffected & \multirow[t]{6}{*}{ Sfânta Elena [31] } \\
\hline & WT 2 & $\begin{array}{l}44^{\circ} 41^{\prime} 37.01^{\prime \prime N} / \\
21^{\circ} 43^{\prime} 9.49^{\prime \prime} \mathrm{E}\end{array}$ & $\begin{array}{l}\text { Grassland } \\
\left(6210^{*}\right)\end{array}$ & $\begin{array}{l}\text { Affected + } \\
\text { Unaffected }\end{array}$ & \\
\hline & WT 4 & $\begin{array}{l}44^{\circ} 41^{\prime} 48.40^{\prime \prime} \mathrm{N} / \\
21^{\circ} 43^{\prime} 7.70^{\prime \prime} \mathrm{E} \\
\end{array}$ & $\begin{array}{l}\text { Grassland } \\
\left(6210^{*}\right)\end{array}$ & $\begin{array}{l}\text { Affected + } \\
\text { Unaffected }\end{array}$ & \\
\hline & WT 9 & $\begin{array}{l}44^{\circ} 41^{\prime} 2.58^{\prime \prime} \mathrm{N} \\
21^{\circ} 44^{\prime} 13.15^{\prime \prime} \mathrm{E}\end{array}$ & $\begin{array}{l}\text { Broad-leaved } \\
\text { forest }\end{array}$ & Unaffected & \\
\hline & WT 21 & $\begin{array}{l}44^{\circ} 41^{\prime} 3.71 " \mathrm{~N} / \\
21^{\circ} 41^{\prime} 48.11^{\prime \prime} \mathrm{E} \\
\end{array}$ & $\begin{array}{l}\text { Grassland }\left(6210^{*}\right) \\
\text { and shrubs }\end{array}$ & Unaffected & \\
\hline & WT 22 & $\begin{array}{l}44^{\circ} 41^{\prime} 16.27^{\prime \prime} \mathrm{N} / \\
21^{\circ} 41^{\prime} 48.79^{\prime \prime} \mathrm{E}\end{array}$ & $\begin{array}{l}\text { Grassland }\left(6210^{*}\right) \\
\text { and shrubs }\end{array}$ & Unaffected & \\
\hline Cirsium grecescui & WT 1 & $\begin{array}{l}44^{\circ} 41 ' 25.62^{\prime \prime N} / \\
21^{\circ} 43^{\prime} 18.09^{\prime \prime} \mathrm{E}\end{array}$ & Arable land & Affected & - \\
\hline
\end{tabular}




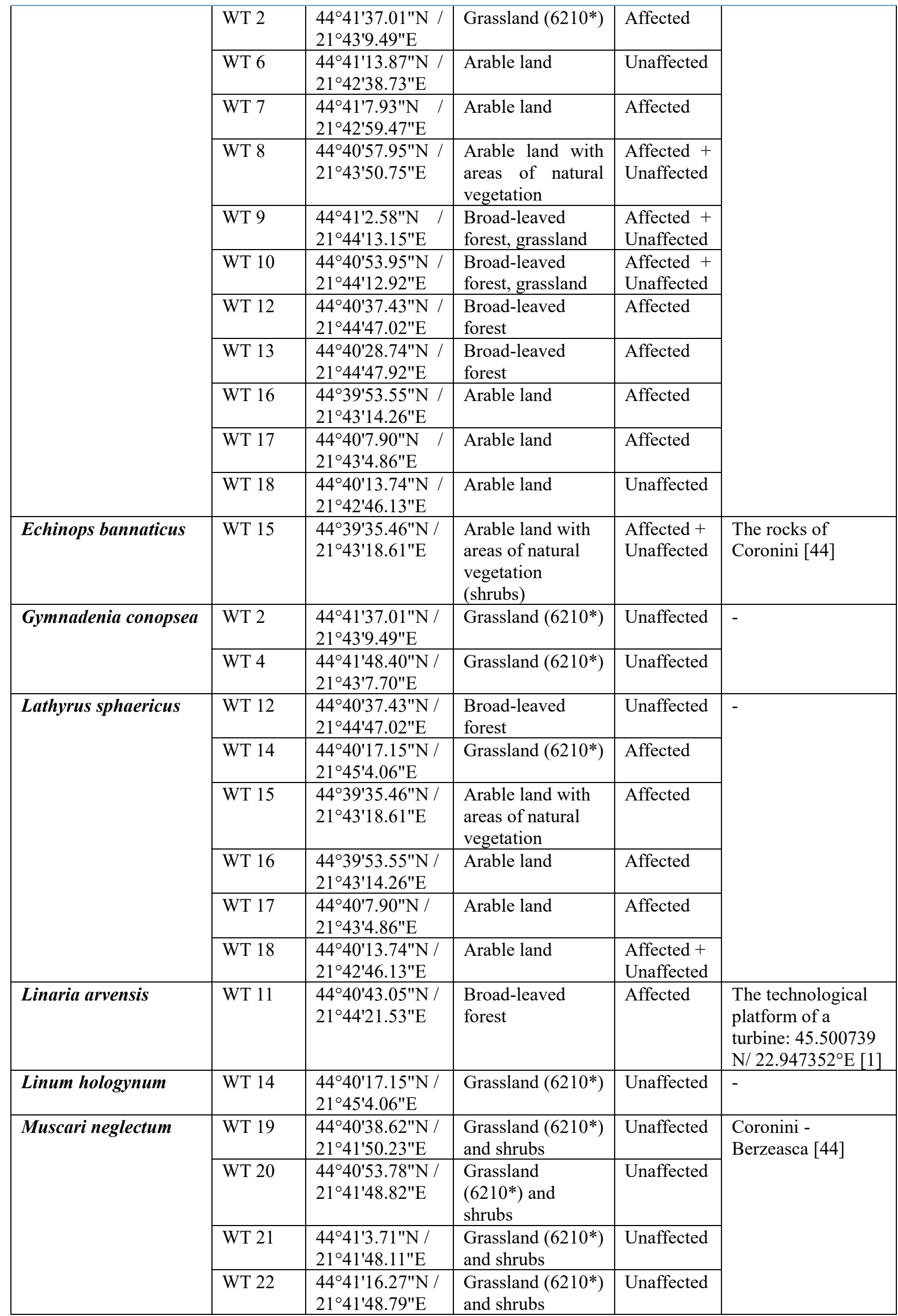




\begin{tabular}{|c|c|c|c|c|c|}
\hline Neotinea ustulata & WT 4 & $\begin{array}{l}44^{\circ} 41^{\prime} 48.40^{\prime \prime} \mathrm{N} / \\
21^{\circ} 43^{\prime} 7.70^{\prime \prime} \mathrm{E}\end{array}$ & Grassland $\left(6210^{*}\right)$ & Unaffected & - \\
\hline Neottia nidus-avis & WT 12 & $\begin{array}{l}44^{\circ} 40^{\prime} 37.43^{\prime \prime} \mathrm{N} / \\
21^{\circ} 44^{\prime} 47.02^{\prime \prime} \mathrm{E}\end{array}$ & $\begin{array}{l}\text { Broad-leaved } \\
\text { forest }\end{array}$ & Unaffected & - \\
\hline Neottia ovata & WT 12 & $\begin{array}{l}44^{\circ} 40^{\prime} 37.43^{\prime \prime} \mathrm{N} / \\
21^{\circ} 44^{\prime} 47.02^{\prime \prime} \mathrm{E}\end{array}$ & $\begin{array}{l}\text { Broad-leaved } \\
\text { forest }\end{array}$ & Unaffected & Sfânta Elena [31] \\
\hline $\begin{array}{l}\text { Orchis mascula subsp. } \\
\text { speciosa }\end{array}$ & $\begin{array}{l}\text { WT } 4- \\
\mathrm{R}>100 \\
\mathrm{~m}\end{array}$ & $\begin{array}{l}44^{\circ} 41^{\prime} 48.40^{\prime \prime} \mathrm{N} / \\
21^{\circ} 43^{\prime} 7.70^{\prime \prime} \mathrm{E}\end{array}$ & Grassland $\left(6210^{*}\right)$ & Unaffected & Sfânta Elena [31] \\
\hline Orchis simia & WT 22 & $\begin{array}{l}44^{\circ} 41^{\prime} 16.27^{\prime \prime} \mathrm{N} / \\
21^{\circ} 41^{\prime} 48.79^{\prime \prime} \mathrm{E} \\
\end{array}$ & $\begin{array}{l}\text { Grassland }\left(6210^{*}\right) \\
\text { and shrubs }\end{array}$ & Unaffected & - \\
\hline \multirow[t]{3}{*}{ Piptatherum virescens } & WT 13 & $\begin{array}{l}44^{\circ} 40^{\prime} 28.74^{\prime \prime} \mathrm{N} / \\
21^{\circ} 44^{\prime} 47.92^{\prime \prime} \mathrm{E}\end{array}$ & $\begin{array}{l}\text { Broad-leaved } \\
\text { forest }\end{array}$ & Unaffected & \multirow{3}{*}{$\begin{array}{l}\text { The rocks of } \\
\text { Coronini, between } \\
\text { Liubcova and } \\
\text { Coronini, Liborăjdii } \\
\text { Valley to Sfânta } \\
\text { Elena [44], } \\
\text { Coronini [35] }\end{array}$} \\
\hline & WT 19 & $\begin{array}{l}44^{\circ} 40^{\prime} 38.62^{\prime \prime} \mathrm{N} / \\
21^{\circ} 41^{\prime} 50.23^{\prime \prime} \mathrm{E}\end{array}$ & $\begin{array}{l}\text { Grassland } \\
\left(6210^{*}\right) \\
\text { and shrubs }\end{array}$ & Unaffected & \\
\hline & WT 20 & $\begin{array}{l}44^{\circ} 40^{\prime} 53.78^{\prime \prime} \mathrm{N} / \\
21^{\circ} 41^{\prime} 48.82^{\prime \prime} \mathrm{E}\end{array}$ & $\begin{array}{l}\text { Grassland }\left(6210^{*}\right) \\
\text { and shrubs }\end{array}$ & Unaffected & \\
\hline Rumex thyrsiflorus & WT 4 & $\begin{array}{l}44^{\circ} 41^{\prime} 48.40^{\prime \prime} \mathrm{N} / \\
21^{\circ} 43^{\prime} 7.70^{\prime \prime} \mathrm{E}\end{array}$ & Grassland $\left(6210^{*}\right)$ & Unaffected & - \\
\hline \multirow[t]{4}{*}{ Smyrnium perfoliatum } & WT 15 & $\begin{array}{l}44^{\circ} 39^{\prime} 35.46^{\prime \prime} \mathrm{N} / \\
21^{\circ} 43^{\prime} 18.61^{\prime \prime} \mathrm{E}\end{array}$ & $\begin{array}{l}\text { Arable land with } \\
\text { areas of natural } \\
\text { vegetation } \\
\text { (shurbs) }\end{array}$ & Unaffected & $\begin{array}{l}\text { The forests between } \\
\text { Gornea and } \\
\text { Coronini [44], } \\
\text { Coronini [49] }\end{array}$ \\
\hline & WT 16 & $\begin{array}{l}44^{\circ} 39^{\prime} 53.55^{\prime \prime} \mathrm{N} / \\
21^{\circ} 43^{\prime} 14.26^{\prime \prime} \mathrm{E}\end{array}$ & Arable land & Unaffected & - \\
\hline & WT 18 & $\begin{array}{l}44^{\circ} 40^{\prime} 13.74 " \mathrm{~N} / \\
21^{\circ} 42^{\prime} 46.13^{\prime \prime} \mathrm{E}\end{array}$ & Arable land & Unaffected & - \\
\hline & WT 21 & $\begin{array}{l}44^{\circ} 41^{\prime} 3.71^{\prime \prime N} / \\
21^{\circ} 41^{\prime} 48.11^{\prime \prime} \mathrm{E}\end{array}$ & $\begin{array}{l}\text { Grassland }\left(6210^{*}\right) \\
\text { and shrubs }\end{array}$ & Unaffected & - \\
\hline Teucrium montanum & WT 20 & $\begin{array}{l}44^{\circ} 40^{\prime} 53.78^{\prime \prime} \mathrm{N} / \\
21^{\circ} 41^{\prime} 48.82^{\prime \prime} \mathrm{E}\end{array}$ & $\begin{array}{l}\text { Grassland }\left(6210^{*}\right) \\
\text { and shrubs }\end{array}$ & Unaffected & $\begin{array}{l}\text { The rocks of } \\
\text { Coronini [44], } \\
\text { Moldova Veche - } \\
\text { Coronini [27] } \\
\text { Coronini - } 1039 \\
\text { mile [30] }\end{array}$ \\
\hline
\end{tabular}

For 11 taxa we assigned Unfavourable-Inadequate conservation status (Agrostemma githago, Anacamptis coriophora, Lathyrus sphaericus, Linum hologynum, Neotinea ustulata, Neottia nidus-avis, Neottia ovata, Orchis simia, Piptatherum virescens, Rumex thyrsiflorus and Teucrium montanum), and only for the species Linaria arvensis did we assign Unfavourable-Bad conservation status. The Unfavourable conservation status was associated with exceedingly small populations, respectively a lower AD score, significant influence of pressure factors, or poor quality of their habitat (e.g., interference with arable or abandoned land, unstable disturbed soil, heavily grazed areas).

Regarding the conservation status for plants with conservation value identified around wind turbines (Table 4), for seven taxa we assigned a Favourable conservation status [11]: Anacamptis morio, Cirsium grecescui, Echinops bannaticus, Gymnadenia conopsea, Muscari neglectum, Orchis mascula subsp. speciosa and Smyrnium perfoliatum. These taxa have numerous individuals in the analysed plots, respectively high AD score, stable populations, large and good quality habitat, and the pressure factors do not affect them significantly. Some of them have recolonized the areas affected by the construction of the wind farm (Fig. 4 A, B). Thus we can 
appreciate that the long-term survival of these species could be ensured, on condition that these areas should be excepted from the maintenance work necessary for the operation of wind turbines.

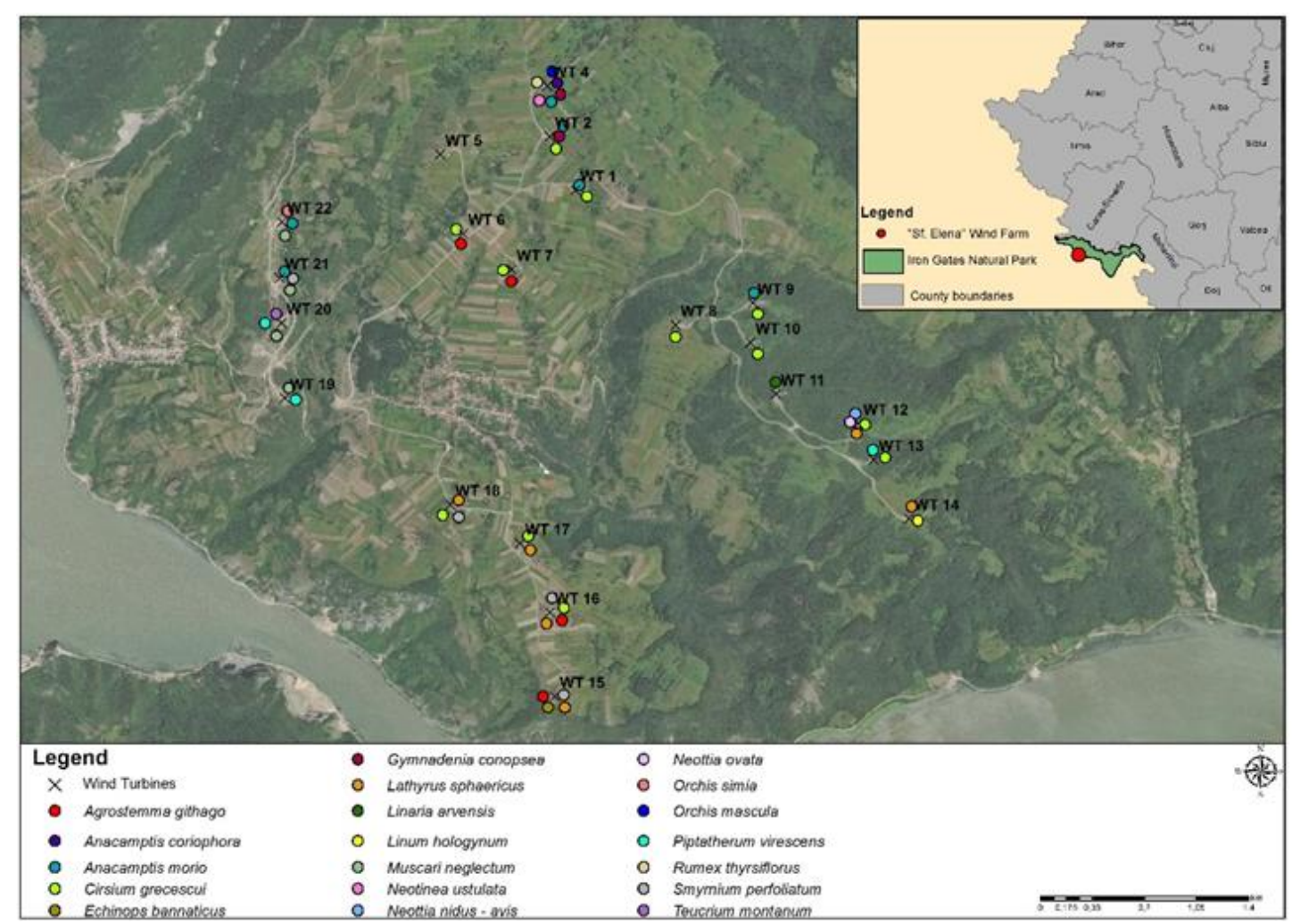

Fig. 2: Distribution of plant taxa with conservation value around the turbines of the "Sfânta Elena" wind farm

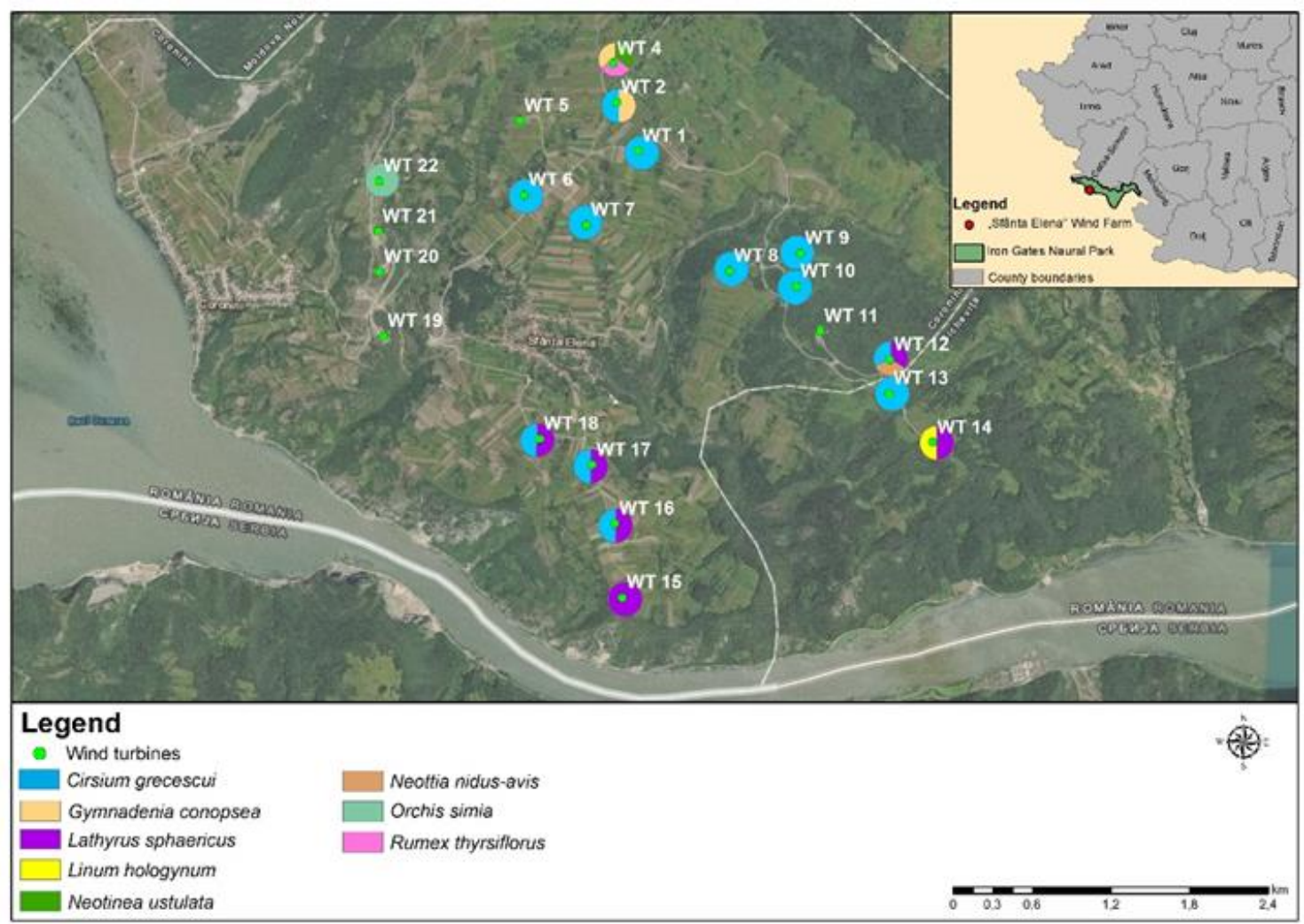

Fig. 3: Distribution of the new reported plant taxa with conservation value for Sfânta Elena Karst Plateau 
Table 3: Frequency of taxa with conservation value in affected and unaffected plots

\begin{tabular}{|l|r|r|}
\hline \multicolumn{1}{|c|}{ Taxa } & $\begin{array}{r}\text { Affected plots } \\
\text { (F\%) }\end{array}$ & $\begin{array}{r}\text { Unaffected plots } \\
\text { (F\%) }\end{array}$ \\
\hline Cirsium grecescui & 47.62 & 23.81 \\
\hline Lathyrus sphaericus & 23.81 & 9.52 \\
\hline Anacamptis morio & 9.52 & 19.05 \\
\hline Agrostemma githago & 14.29 & 9.52 \\
\hline Muscari neglectum & 0.00 & 19.05 \\
\hline Smyrnium perfoliatum & 0.00 & 19.05 \\
\hline Piptatherum virescens & 0.00 & 14.29 \\
\hline Echinops bannaticus & 4.76 & 4.76 \\
\hline Gymnadenia conopsea & 0.00 & 9.52 \\
\hline Linum hologynum & 0.00 & 4.76 \\
\hline Anacamptis coriophora & 0.00 & 4.76 \\
\hline Linaria arvensis & 4.76 & 0.00 \\
\hline Neotinea ustulata & 0.00 & 4.76 \\
\hline Neottia nidus-avis & 0.00 & 4.76 \\
\hline Neottia ovata & 0.00 & 4.76 \\
\hline Orchis mascula subsp. speciosa & 0.00 & 4.76 \\
\hline Orchis simia & 0.00 & 4.76 \\
\hline Rumex thyrsiflorus & 0.00 & 4.76 \\
\hline Teucrium montanum & 0.00 & 4.76 \\
\hline
\end{tabular}

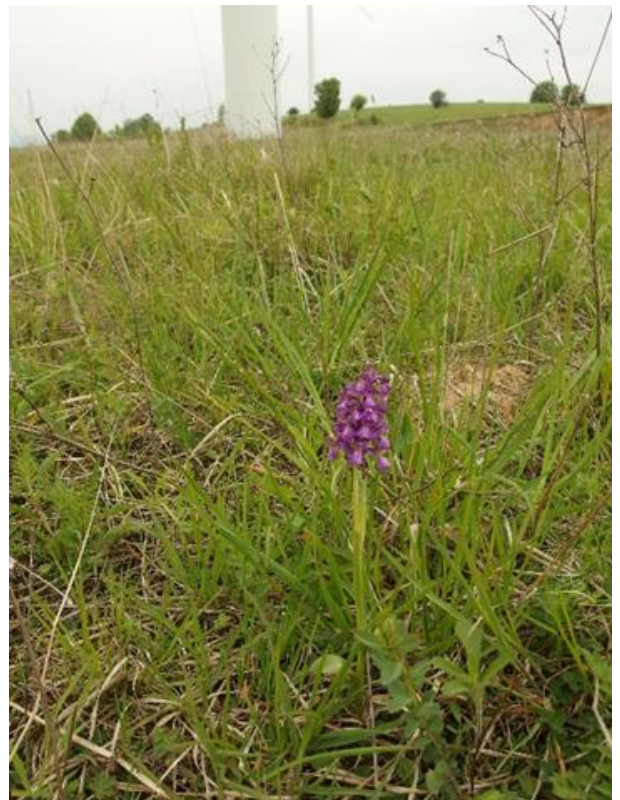

(A)

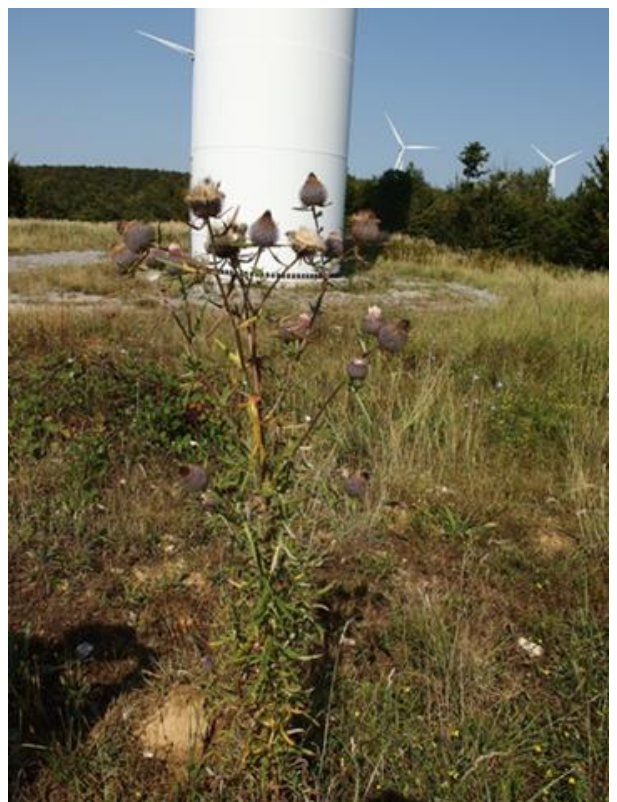

(B)

Fig. 4: Plants with conservation value observed on the affected areas (technological platforms); (A) Anacamptis morio; (B) Cirsium grecescui 
Table 4: Conservation status for the plants with conservation value around wind turbines

\begin{tabular}{|c|c|c|c|}
\hline Taxa name & AD & Pressures & Conservation status \\
\hline Agrostemma githago & $\mathbf{r}$ & Weed control & $\begin{array}{l}\text { Unfavourable - } \\
\text { Inadequate }\end{array}$ \\
\hline Anacamptis coriophora & $\mathbf{r}$ & $\begin{array}{c}\text { Invasion of Erigeron annuus subsp. annuus } \\
\text { Grazing }\end{array}$ & $\begin{array}{l}\text { Unfavourable - } \\
\text { Inadequate }\end{array}$ \\
\hline Anacamptis morio & 2 & $\begin{array}{c}\text { Invasion of Erigeron annuus subsp. annuиs } \\
\text { Grazing }\end{array}$ & Favourable \\
\hline Cirsium grecescui & 1 & - & Favourable \\
\hline Echinops bannaticus & 2 & Invasion of Erigeron annuus subsp. annuиs & Favourable \\
\hline Gymnadenia conopsea & 1 & Invasion of Erigeron annuus subsp. annuus & Favourable \\
\hline Lathyrus sphaericus & + & $\begin{array}{c}\text { Poor habitat quality (ruderalized } \\
\text { grasslands, areas with disturbed soil) }\end{array}$ & $\begin{array}{l}\text { Unfavourable - } \\
\text { Inadequate }\end{array}$ \\
\hline Linaria arvensis & $\mathbf{r}$ & Removing vegetation from platforms & Unfavourable - Bad \\
\hline Linum hologynum & + & \begin{tabular}{|c|} 
Invasion of Erigeron annuus subsp. annuиs \\
Expansion of woody vegetation
\end{tabular} & $\begin{array}{l}\text { Unfavourable - } \\
\text { Inadequate }\end{array}$ \\
\hline Muscari neglectum & 2 & Grazing & Favourable \\
\hline Neotinea ustulata & $\mathbf{r}$ & Invasion of Erigeron annuus subsp. annuиs & $\begin{array}{l}\text { Unfavourable - } \\
\text { Inadequate } \\
\end{array}$ \\
\hline Neottia nidus-avis & $\mathbf{r}$ & - & $\begin{array}{l}\text { Unfavourable - } \\
\text { Inadequate }\end{array}$ \\
\hline Neottia ovata & $\mathbf{r}$ & - & $\begin{array}{c}\text { Unfavourable - } \\
\text { Inadequate }\end{array}$ \\
\hline $\begin{array}{c}\text { Orchis mascula subsp. } \\
\text { speciosa }\end{array}$ & 2 & - & Favourable \\
\hline Orchis simia & $\mathbf{r}$ & $\begin{array}{l}\text { The presence of the invasive species } \\
\text { Ailanthus altissima }\end{array}$ & $\begin{array}{l}\text { Unfavourable - } \\
\text { Inadequate }\end{array}$ \\
\hline Piptatherum virescens & + & $\begin{array}{l}\text { The presence of the invasive species } \\
\text { Ailanthus altissima }\end{array}$ & $\begin{array}{l}\text { Unfavourable - } \\
\text { Inadequate } \\
\end{array}$ \\
\hline Rumex thyrsiflorus & $\mathbf{r}$ & $\begin{array}{c}\text { Poor habitat quality (boundary arable land } \\
\text { with grassland) }\end{array}$ & $\begin{array}{l}\text { Unfavourable - } \\
\text { Inadequate }\end{array}$ \\
\hline Smyrnium perfoliatum & 1 & - & Favourable \\
\hline Teucrium montanum & + & $\begin{array}{l}\text { Poor habitat quality (areas with disturbed } \\
\text { soil) }\end{array}$ & $\begin{array}{l}\text { Unfavourable - } \\
\text { Inadequate } \\
\end{array}$ \\
\hline
\end{tabular}

The orchid species Anacamptis morio, Gymnadenia conopsea, Orchis mascula subsp. speciosa, Anacamptis coriophora, and Neotinea ustulata have been identified in grassland habitats, while the other three orchids have been identified in forest areas (Neottia nidus-avis and Neottia ovata), or in scrub vegetation associated with grasslands (Orchis simia). The species with the most individuals identified in grassland habitats are as follows: Anacamptis morio (over 100), and Gymnadenia conopsea (over 30), Orchis mascula subsp. speciosa (about 20). The populations of the other two orchid species (Neotia nidus-avis and Neotinea ustulata) are reduced to no more than two individuals. The pressure factors on the orchid species are those acting on their habitats: expansion of arable land in the grassland area, the presence of invasive plants like Erigeron annuиs subsp. annuus and Ailanthus altissima, grazing (goat herds are frequently observed in the area), and also the tourist activity that has become very intense in the area (ATV rides are practiced even on grasslands).

Orchid grasslands at the wind farm site correspond to Natura 2000 habitat $6210^{*}$ Seminatural dry grasslands and scrubland facies on calcareous substrates (Festuco-Brometea) (* 
important orchid sites) that has been identified around five turbines (WT 2, WT 4, WT 14, WT 21, and WT 22) (Fig. 5). The habitat consists of dry or semi-dry grasslands on calcareous substrate which are often in association with scrub and thermophilic forests and contains many species of the Festuco-Brometea class, but also various species of orchid [24, 36]. In the area of the wind farm this type of habitat covers an area quite small, being interspersed between the forests and arable land.

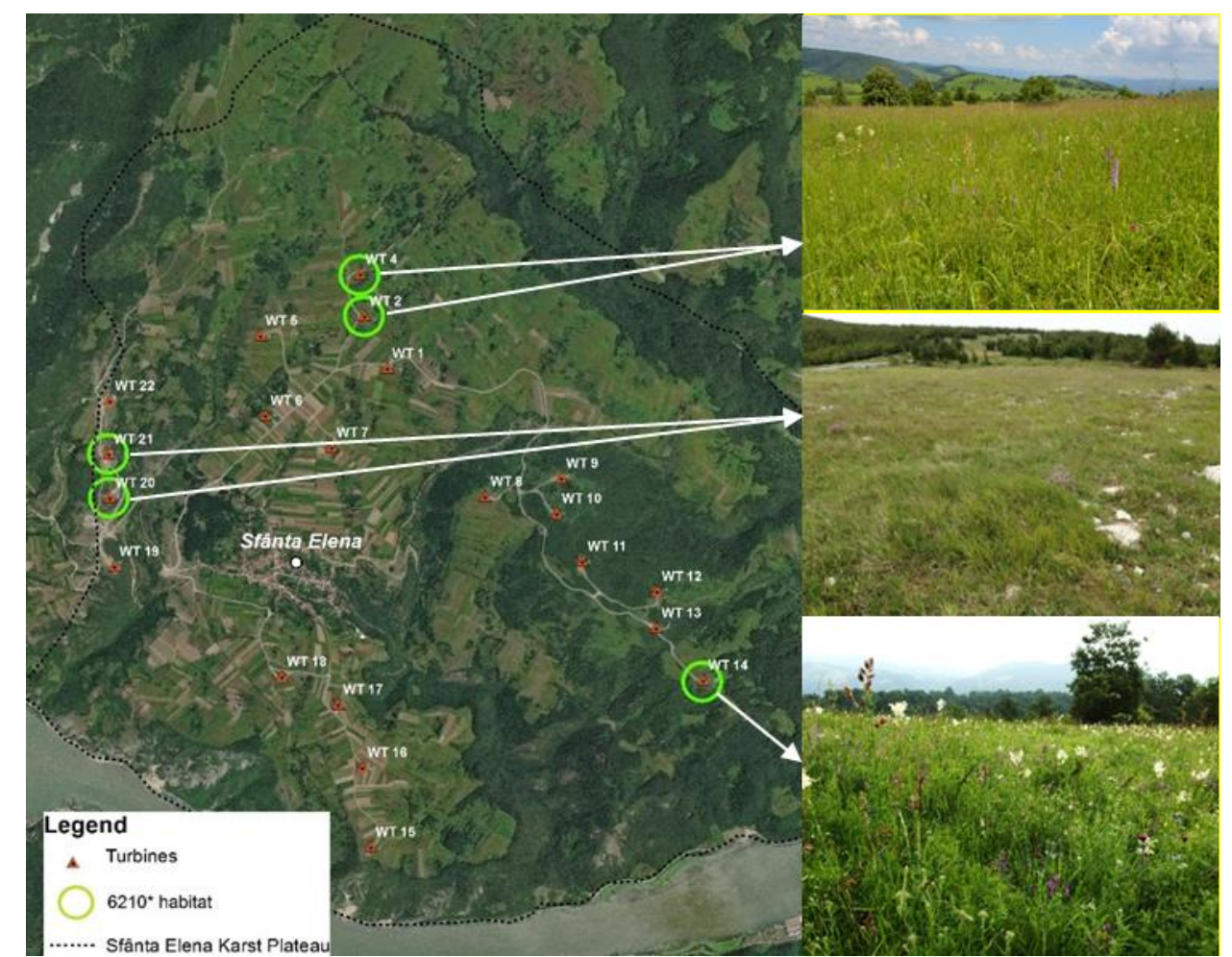

Fig. 5: Locations of plots with $6210^{*}$ habitat and their appearance around wind turbines of "Sfânta Elena" wind farm

The analysis of the structure and floristic composition of this habitat from the grasslands around wind turbines, by comparison with the criteria of the Favourable conservation status [36], showed us that the habitat falls within the acceptable limits, as follows:

- The total area of the habitat $6210 *$ in the five plots of unaffected habitat is 1.25 ha, which represents $0.94 \%$ of the total area of the habitat on the entire site Iron Gates Natural Park (133 ha). In these five plots, we inventoried 229 vascular plant taxa and the species richness varies between 86 and 113 taxa within the five plots. Thus, the species richness significantly exceeds the minimum of 20 plant species needed to qualify under Favourable conditions according to Habitat fact-sheets [36].

- The dominant and characteristic plant species in the plots around wind turbines correspond to $46.87 \%$ of the total species mentioned by Mountford et al. [36], namely: Anthyllis vulneraria, Bothriochloa ischaemum, Brachypodium pinnatum, Campanula glomerata, Centaurea scabiosa, Dianthus carthusianorum, Festuca valesiaca, Asperula cynanchica, Medicago falcata, Anacamptis coriophora, Anacamptis morio, Neotinea ustulata, Orlaya grandiflora, Potentilla argentea, Sanguisorba minor. Some other species which identified the floristic composition of the habitat around the five turbines are: Briza media, Calamagrostis epigejos, Campanula patula 
subsp. patula, Campanula persicifolia, Dactylis glomerata, Festuca pratensis, Filipendula vulgaris, Genista tinctoria, Gymnadenia conopsea, Helianthemum nummularium subsp. nummularium, Koeleria macrantha, Melica ciliata, Muscari neglectum, Orchis simia, Pimpinella saxifraga, Polygala comosa, Rhinanthus rumelicus, Salvia verticillata, Scabiosa ochroleuca, Stachys officinalis, Teucrium chamaedrys, Thymus pulegioides subsp. pannonicus, Trifolium montanum, Vicia cracca, Xeranthemum annuum, etc.

- There is an impressive number of rare plant species in the plots around the turbines, many being represented by orchids: Anacamptis morio, Gymnadenia conopsea, Neotinea ustulata, Anacamptis coriophora, Orchis simia, Smyrnium perfoliatum and Linum hologynum.

- Although Mountford et al. [36] do not mention the presence of species with negative impact (alien plants) for the 6210* habitat, we found around wind turbines the invasive alien species Ailanthus altissima, Erigeron canadensis, Erigeron annuus subsp. annuus and Robinia pseudoacacia. The species Ailanthus altissima and Robinia pseudoacacia are present at the interface of grasslands with woody vegetation (hornbeam scrub) (WT 21 and WT 22). The presence of Erigeron annuus subsp. annuus and Erigeron canadensis can be explained by the fact that the grasslands are fragmented by cultivated or abandoned arable lands that are areas suitable for alien species. Also, the areas covered with the $6210^{*}$ habitat are grazed by herds of goats and cows, which to a certain extent may be a factor of degradation of the vegetation structure and composition.

- The height of the vegetation corresponds to the limits of 20-100 cm described by Mountford et al. [36]. The maximum height is denoted by the species Brachypodium pinnatum, Briza media, Bothriochloa ischaemum, Calamagrostis epigejos, Dianthus carthusianorum, Festuca valesiaca, etc.

- The grassland areas are mixed with mosaics of scrub vegetation and trees, as described by Mountford et al. [36]. Around WT 21 and WT 22, the grassland habitat intersects with Carpinus orientalis scrub, including some individuals of Quercus pubescens, Crataegus monogyna and Rosa canina. Scattered individuals of the invasive species Ailanthus altissima and Robinia pseudoacacia are present at the edge of the hornbeam bushes. Around WT 14, WT 2 and WT 4, scattered shrubs and trees are present, such as: Corylus avellana, Populus tremula, Rosa gallica, Tilia tomentosa, Sorbus torminalis, Pyrus communis subsp. pyraster, Quercus cerris, Quercus pubescens and Rosa canina.

- The vegetation of the habitat $6210^{*}$ consists of mostly perennial species; the plant associations are at the climax stage. In the case of the plot from the WT 14, the extension of the juveniles of downy oak (Quercus pubescens) has been observed.

The negative effects of the wind farm on the orchid-rich grasslands were manifested mainly during the construction period, involving the removal and destruction of vegetation for the construction of various facilities. Some land areas, such as turbine foundations and access roads have remained permanently in use in the study area, which means that those surfaces are permanently lost in terms of vegetation cover during the operation of the wind farm, and the vegetation will not recover in the missing of the decommissioning workings. However, other affected land areas during the construction phase, such as technological platforms, were temporarily cleared of vegetation, and after construction stage they were naturally recovered with vegetation. After about 10 years from the construction of the wind farm, the habitats in the immediate vicinity, unaffected by construction workings, have maintained their structure and 
floristic composition described in the literature and are within the limits of Favourable conditions as described by Mountford et al. [36].

In the studied area there are other impact factors that can have a higher negative effect on grasslands as compared to wind farms (e.g., invasive alien species, intensive grazing, conversion of grassland to arable land, the burning of stubble by the inhabitants of the area, tourism, etc.). Most of the invasive alien species recorded in the analysed plots have a common presence in the area, unrelated to the construction of the wind farm. Thus, the presence of invasive woody species Ailanthus altissima derives from the plantations in the Iron Gate Natural Park where it was used for ornamental purposes [26]. The presence of herbaceous species such as Erigeron canadensis and Erigeron annus subsp. annuus is associated with the presence of cultivated or abandoned arable land that either interdigitates with grassland habitats or is located in the immediate vicinity. Intensive grazing represents a strong pressure on the habitats in the area, followed by the presence and activities of tourists. As a result of tourism there is waste (especially plastics like PET, bags, etc.), burnt debris from grills made at the edge of the forest, and the entry of ATVs into grassland habitats. Like the observations of Fagúndez [21], we found that facilitating access to the area allowed the growth of human activities in protected areas, such as grazing, road traffic, searching and collecting plants etc. Although roads and restoration activities are known in the literature as a source of habitat colonization with pioneer and invasive species [23, 42, 54], in the case of grasslands around "Sfânta Elena" wind farm, the presence of alien invasive species was related to other anthropogenic factors manifested in the area (plantations of non-native species or interference with arable or abandoned land).

As Silva and Passos [54] show, an obstacle in estimating the impact of wind farms on natural and semi-natural habitats is the lack of floristic data prior to construction. In the case of the SEKP, the plant species distribution and vegetation data from scientific literature are insufficient for estimating the real impact of the wind turbines. There is also a need for studies outside the wind farm limits, as it is obvious that there are still interesting undiscovered floristic elements in the territory of the SEKP. Moreover, it is necessary to conduct complex studies to assess the cumulative impact of negative pressure factors and to outline effective measures for the conservation of natural habitats present in this territory.

\section{Conclusions}

The conservation status of plants and grasslands with conservation value on SEKP is correlated with the various anthropogenic pressures in the area. The operation activities of the wind farm are reduced to the existing infrastructure and the nearby vegetation are not affected.

Insufficient floristic data in the area of the SEKP before the construction of wind turbines (e.g. taxon distribution, population size, habitat mapping and their structural and composition descriptions) represent an impediment in accurately estimating the impact of wind turbines. Further research is needed, outside the site of the wind farm, for a complete image of the floristic elements with conservative value of the SEKP.

Acknowledgements: We express our gratitude to Enel Green Power Romania for agreeing to provide access to the wind farm. We also thank the Iron Gates Natural Park Administration for agreeing this research in the Iron Gates Natural Park. We are very grateful to Dr. Gavril Negrean who helped us find the scientific literature and clarify some taxonomical aspects. We thank the geographer Claudia Tomescu who helped us to elaborate the distribution maps in the ArcGIs software. 


\section{REFERENCES}

1. Anastasiu, P., 2015, New chorological data for rare vascular plants from Romania, Acta Horti Botanici Bucurestiensis, 42 (1): 57-62. DOI: 10.1515/ahbb-2015-0002.

2. Beldie, A., 1952, Fam. Fagaceae. In: Săvulescu, T. (red. pr.), Flora Republicii Populare Române, Vol. 1 (pp. 217 260), Editura Academiei Române, București.

3. Borza, A., 1931, Botanischer führer durch die umgebung von Băile Herculane (Herkulesbad) bis an die Donau, [Botanical guide about surroundings of Băile Herculane area to the Danube]. In: Borza, A. (ed.), Guide de la sixième excursion phytogéographique internationale, Roumanie, [Guide of the Sixth International Phytogeographic Excursion, Romania] (pp. 55-63), Le jardin botanique de L’Université de Cluj, Cluj

4. Boşcaiu, N., Coldea, G., Horeanu, C., 1994, Lista Roşie a plantelor vasculare dispărute, periclitate, vulnerabile şi rare din flora României [Red List of extinct, endangered, vulnerable and rare vascular plants in Romania's flora], Ocrotirea Naturii şi a Mediului Înconjurător, 38 (1): 45-56.

5. Călinescu, R., Iana, S., 1964, Considerațiuni biogeografice asupra Defileului Dunării [Biogeographical considerations on the Danube Gorge], Analele Universității din București, Științele Naturii Geologie-geografie, 13 (1): 151-168.

6. Capmourteres, V., Anand, M., 2016, "Conservation value": a review of the concept and its quantification. Ecosphere, 7 (10): e01476. DOI: 10.1002/ecs2.1476.

7. Ciortan, I., Negrean, G., 2012, Rumex thyrsiflorus in Romania, Analele Universității din Craiova, seria Biologie, Horticultură, Tehnologia Prelucrării Produselor Agricole, Ingineria Mediului, 17 (53): 603-608.

8. Coldea, G., Boşcaiu, N., Lupşa, V.P.E, Resmeriţă, I., 1970, Vegetaţia făgetelor din sectorul Valea Eşelniţa-Valea Mraconiei al Defileului Dunării [Beech vegetation in the Eşelniţa Valley - Mraconia Valley sector of the Danube Gorge], Studii și cercetări de biologie, Seria Botanică, 22 (6): 467-474.

9. Comănescu, P, Conţeanu, M., Nagodă, E., 2020, Threatened vascular plants from BUC Herbarium (I), Acta Horti Botanici Bucurestiensis, 46: 55-105.

10. Comănescu, P., Stefănuț, S., 2011, The chorology of the species Sesleria rigida and Sesleria filifolia in Romania. Acta Horti Botanici Bucurestiensis, 38: 39-52.

11. Combroux, I., Schwoerer, C., 2007, Evaluarea statului de conservare al habitatelor și speciilor de interes comunitar din România - ghid methodologic [Assessment of the conservation status of habitats and species of community interest in Romania - methodological guide], Editura Balcanic, Timişoara.

12. Commission of the European Community, 2013, Interpretation manual of European Union habitats-EUR 28. European Commission DG Environment, Brussels.

13. Coste, I., 1974, Flora şi vegetaţia Munţilor Locvei [Flora and vegetation of the Locvei Mountains], PhD thesis, "Babeş-Bolyai" University Cluj-Napoca.

14. Council of the European Communities, 1992, Council Directive 92/43/EEC of 21 May 1992 on the conservation of natural habitats and of wild fauna and flora. Official Journal of the European Communities L206/7.

15. Dihoru, G., Negrean, G., 2009, Cartea roşie a plantelor vasculare din România, [Romanian Red Book of Vascular Plants], Editura Academiei Române, Bucureşti.

16. Dihoru, G., Dihoru, A., 1994, Plante rare, periclitate şi endemice în Flora României - Lista roşie, [Rare, endangered and endemic plants of Romania's Flora - The Red List], Acta Horti Botanici Bucurestiensis, 19931994: 173-197

17. Dobrescu, C., 1972, Genul Cleistogenes Keng. In Săvulescu, T. (red. pr.). Flora Republicii Socialiste România, Vol 12 (pp. 354-365), Editura Academiei Române, București.

18. Eliáš, P. jun, Eliáš, P. sen, Baranec, T., 2007, The New red list of Slovak endangered weeds. In: Eliáš P jun (Ed), Threatened weedy plant species. Book of proceedings from the international conference, pp. 23-28, Slovak University of Agriculture, Nitra.

19. Esri, 2016, ArcGIS advanced: realease 10.4. Redlands, CA: Environmental Systems Research Institute.

20. Euro+Med (2006-): Euro+Med PlantBase - the information resource for Euro-Mediterranean plant diversity. Retrieved 2020 September 30 from: http://ww2.bgbm.org/EuroPlusMed/

21. Fagúndez, J., 2008, Effects of wind farm construction and operation on mire and wet heath vegetation in the Monte Maior SCI, north-west Spain, Mires \& Peat, 4 (2): 1-12. 
22. Firbank, L. G., 1988, Agrostemma githago L. (Lychnis githago (L.) Scop.), Journal of Ecology, 76 (4): 12321246.

23. Fraga, M.I, Romero-Pedreira, D, Souto, M, Castro, D, Sahuquillo, E., 2008, Assessing the impact of wind farms on the plant diversity of blanket bogs in the Xistral Mountains (NW Spain), Mires and Peat, 4 (6): 1-10.

24. Gafta, D., Mountford, O., Alexiu, V., Anastasiu, P., Bărbos., M., Burescu, P., Coldea, G., Drăgulescu, C., Făgăraş, M., Goia, I., Groza, G., Micu, D., Mihăilescu, S., Moldovan, O., Nicolin, A., Niculescu, M., Oprea, A., Oroian, S., Paucă-Comănescu, M., Sârbu, I., Şuteu, A., 2008, Manual de interpretare a habitatelor Natura 2000 din România [Interpretation manual of Natura 2000 habitats in Romania], Editura Rosprint, Cluj-Napoca.

25. Ghișa, E., 1972, Genul Chrysopogon Trin. In: Săvulescu T. (red. pr.). Flora Republicii Socialiste România, Vol 12 (pp. 53-57), Editura Academiei Român, București.

26. Goia, I., Ciocanea, C.M, Gavrilidis, A.A., 2014, Geographic origins of invasive alien species in "Iron Gates" Natural Park (Banat, Romania), Transylvanian Review of Systematical and Ecological Research, 16: 115-130. DOI:10.1515/trser-2015-0036.

27. Grigore, S., Coste, I., 1978, Cercetări asupra vegetației dintre Moldova Veche și Pescari (județul Caraş-Severin) [Vegetation research between Moldova Veche and Pescari (Caraş-Severin county)], Banatica, Caiete de Stiinte naturale, Studii și Cercetări de Geologie, Geografie, Biologie Reșița, 7: 173-189.

28. Grințescu, I., 1958, Fam. Vitaceae. In: Săvulescu T. (red. pr.). Flora Republicii Populare Române, Vol 6 (pp. 283 -316), Editura Academiei Române, București.

29. Hamanová, M., 2013, Wind Farms and Tourism in The Romanian Banat. In: Fialová J, Kubičcová H (eds), Public recreation and landscape protection - with man hand in hand...Conference proceeding 1st - 3rd May 2013, Brno: Publishing Centre Mendel University in Brno pp 221-224.

30. Matacă., S.Ş., 2005, Parcul Natural Porțile de Fier. Floră, vegetație și protecția naturii [Iron Gates Natural Park. Flora, vegetation and nature protection], Editura Universitaria, Craiova.

31. Milanovici, S., 2012, Orhidee din sudul Banatului (Orchids from south-west side of Banat region), East-West Print, Timișoara.

32. Moisi, Al., 1938, Monografia Clisurii [Monograph of the Danube Strait], Felix Weiss, Oraviţa.

33. Morariu, I., 1957, Genul Circaea L.. In: Săvulescu, T. (red. pr.). Flora Republicii Populare Române, Vol. 5 (pp. 519-525), Editura Academiei Române, București.

34. Morariu, I., 1972, Genul Cynosurus L.. In: Săvulescu, T. (red. pr.). Flora Republicii Socialiste Române, Vol 12 (pp. 338-341), Editura Academiei Române, București.

35. Morariu, I., 1972, Genul Oryzopsis L.. In: Săvulescu T. (red. pr.). Flora Republicii Socialiste Române, Vol 12 (pp. 186-190), Editura Academiei Române, București.

36. Mountford, O., Gafta, D., Anastasiu, P., Bărbos, M.I., Nicolin, A., Niculescu, M., Oprea, A., 2008, Natura 2000 in Romania: Habitat fact-sheets, Romanian Ministry of Environment and Sustainable Development.

37. Negrean, G., Karácsonyi, C., Szatmari, P.M., 2017. General description of the Sălaj flora. Contributii Botanice, 52: 7-21.

38. Nyárády, E.J., 1956, Genul Rubus L. In: Săvulescu T. (red. pr.). Flora Republicii Populare Române, Vol. 4 (pp. 276-580), Editura Academiei Române, București.

39. Oancea, D., Velcea, V., Caloianu, N., Dragomirescu, Ș., Dragu, Gh., Mihai, E., Niculescu, Gh., Sencu, V., Velcea, I., 1987, Geografia României, Vol. 3 - Carpații Românești și Depresiunea Transilvaniei [Geography of Romania, Vol. 3 - The Romanian Carpathians and the Transylvanian Depression], Editura Academiei Române, București.

40. Oltean, M., Negrean, G., Popescu, A., Roman, N., Dihoru, G., Sanda, V., Mihăilescu, S., 1994, Lista roşie a plantelor superioare din România, [Red List of superior plants in Romania]. In: Oltean M. (coord), Studii, sinteze, documentaţii de ecologie, Academia Română, Institutul de Biologie, Bucureşti.

41. Paşcovschi, S., Leandru, V., 1958, Tipuri de pădure din Republica Populară Romînă, Vol. 14. Editura AgroSilvică de Stat, București.

42. Passos, I., Silva, M., Mesquita, S., Marques, A.T., Bernardino, J., Mascarenhas, M., 2013, Aliens in wind farms preventing and monitoring impacts on vegetation, [Abstract]. In: Conference on wind power and environmental impacts, Naturvårdsverket rapport pp 80.

43. Paucă, A., 1958, Fam Anacardiaceae. In: Săvulescu, T. (red. pr.). Flora Republicii Populare Române, Vol 6 (pp. 214-219), Editura Academiei Române, București.

44. Păun, M., Cîrțu, M., Cîrțu, D., Popescu, G., 1968, Materiale pentru flora dintre Berzasca şi Pescari, Judeţul Caraş-Severin, Buletin Ştiinţific Craiova, 10: 47-62. 
45. Păun, M., Popescu, G., Cîrțu, M., Cîrțu, D., 1970, Aspecte din vegetația teritoriului dintre Berzeasca şi Pescarii (Jud. Caraș-Severin), Analele Universităţii Craiova Seria Biologie- Ştiinţe Agricole, 2 (12): 61-70.

46. Pop, O., 2001, Distributon of Asplenium lepidum and Asplenium ruta-muraria ssp. ruta-muraria in România, Acta Horti Botanici Bucurestiensis, 29: 139-147.

47. Prodan, I., 1953, Genul Cerastium L.. In: Săvulescu, T. (red. pr.). Flora Republicii Populare Române, Vol. 2 (pp. 39-65), Editura Academiei Române, București.

48. Prodan, I., Nyárády, E.J., 1964, Genul Centaurea L.. In: Săvulescu T. (red. pr.). Flora Republicii Socialiste Române, Vol. 9 (pp. 785-951), Editura Academiei Române, București.

49. Sandu-Ville, C., Eliade, E., Comes, I., Iacob, V., Manoliu, A., Mititiuc, M., Rusan, M., Toma, M., Gutu, E., Ene, I., Costescu, M., Glodeanu, C., 1973, Micoflora din zona sistemului Hidroenergetic și de navigație "Porțile de Fier" [Mycoflora in the area of the Hydropower and navigation system "Iron Gates], Acta Botanica Horti Bucurestiensis, 1972-1973: 579-645.

50. Šantrůčková, M., Pákozdiová, M., Hamanová, M., 2014, Local community versus globalization tendencies: Case study of Czech villages in Romanian Banat Region, Journal of Landscape Ecology, 7 (2):73-89. DOI:10.2478/jlecol-2014-0017

51. Sârbu, I., Ştefan, N., Oprea, A., 2013, Plante vasculare din România. Determinator ilustrat de teren, Editura Victor B Victor, București.

52. Šebesta, J., Maděra, P., Řepka, R., Matula, R., 2017, Comparison of vascular plant diversity and species composition of coppice and high beech forest in the Banat region, Romania, Folia Geobotanica, 52: 33-43. DOI: 10.1007/s12224-016-9279-3

53. Sencu, V., 1972, Carstul din Defileu [The Gorge Carst]. In: Vulcănescu, R. (coord.), Atlasul Complex "Porțile De Fier" (pp 151-152), Editura Academiei Române, București,

54. Silva, M.R., Passos, I., 2017, Vegetation. In: Perrow, M.R., (ed.), Wildlife and wind farms, conflicts and solutions, Vol. 1 Onshore: potential effects (pp 40-62), Pelagic Publishing, Exeter, UK.

55. Sîrbu, C., Oprea, A., 2011, Plante adventive în flora României [Alien plants in the Romanian flora], Editura "Ion Ionescu de la Brad", Iași.

56. Št astná, M., Vaishar, A., Pákozdiová, M., 2015, Role of tourism in the development of peripheral countryside. Case studies of Eastern Moravia and Romanian Banat, Forum Geografic, 14 (1): 83-93. DOI: 10.5775/fg.20674635.2015.198.i

57. Szatmari, P-M., 2015, Additional Glacial Relicts in Carei Plain natural protected area, north-western Romania, Acta Horti Botanici Bucurestiensis, 42: 23-40.

58. Todor, I., 1958. Fam. Umbeliferae. In: Săvulescu, T. (red. pr.), Flora Republicii Populare Române, Vol. 6. (pp. 326-652), Editura Academiei Române, București.

59. Trnka, F., Stejskal, R., Skuhrovec, J., 2016, The morphology of the immature stages of two rare Lixus species (Coleoptera, Curculionidae, Lixinae) and notes on their biology, ZooKeys, 604: 87-116. DOI: 10.3897/zookeys.604.9018.

\section{STAREA DE CONSERVARE A PLANTELOR ȘI HABITATELOR DUPĂ 10 ANI DE FUNCȚIONARE A UNUI PARC EOLIAN. STUDIU DE CAZ: PLATOUL CARSTIC SFÂNTA ELENA, PARCUL NATURAL PORȚILE DE FIER, ROMANIA}

\section{(Rezumat)}

Localizat în Parcul Natural Porțile de Fier, România, la poalele Munților Locvei, Platoul Carstic Sfânta Elena este un teritoriu foarte pitoresc, caracterizat de un relief carstic, acoperit de pajişti de mare valoare conservativă. În ultimul deceniu, acest teritoriu a fost supus unor presiuni antropice în creștere, iar îngrijorările cele mai mari asupra biodiversității au apărut odată cu ridicarea unui parc eolian în anul 2011. Pornind de la o îngrijorare exprimată de un cercetător român, studiul nostru își propune să furnizeze date actuale privind starea de conservare a speciilor de plante cu valoare conservativă și a pajiștilor cu orhidee din jurul turbinelor eoliene, după zece ani ai fazei operaționale. În această perioadă am identificat 19 taxoni cu valoare conservativă, dintre care opt specii de orhidee. Patru dintre orhidee fac obiectul unor noi raportări privind flora acestui teritoriu: Gymnadenia conopsea, Neotinea ustulata, Neottia nidusavis și Orchis simia, alături de alți taxoni care sunt menționați pentru prima dată, precum Cirsium grecescui, Lathyrus 
sphaericus, Linum hologynum și Rumex thyrsiflorus. Pajiștile cu orhidee din zona parcului eolian corespund habitatului Natura 2000 6210* a cărui structură și compoziție floristică se încadrează în limitele acceptabile ale stării de conservare Favorabile. Starea de conservare a speciilor de plante depinde în mod direct de calitatea habitatului lor, precum și de varietatea factorilor de presiune și amenințare din zonă.

Received: 7.09.2021; Accepted: 30.09.2021. 